\title{
14-3-3 $\zeta$ Regulates Immune Response through Stat3 Signaling in Oral Squamous Cell Carcinoma
}

\author{
Xinguang Han*, Yongfu Han, Huifeng Jiao, and Yaqiong Jie ${ }^{\top}$
}

\begin{abstract}
Ectopic expression of 14-3-3ל has been found in various malignancies, including lung cancer, liver cancer, head and neck squamous cell carcinoma (HNSCC), and so on.

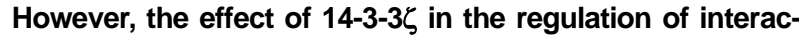
tions between tumor cells and the immune system has not been previously reported. In this study, we aimed to investigate whether and how 14-3-3C is implicated in tumor inflammation modulation and immune recognition evasion. In oral squamous cell carcinoma (OSCC) cell lines and cancer tissues, we found that 14-3-3 $\zeta$ is overexpressed. In OSCC cells, 14-3-35 knockdown resulted in the up-regulated expression of inflammatory cytokines. In contrast, 14-3-3 $\zeta$ introduction attenuated cytokine expression in human normal keratinocytes and fibroblasts stimulated with interferon- $\gamma$ (IFN- $\gamma$ ) and lipopolysaccharide (LPS). Furthermore, supernatants from 14-3-3ל knockdown OSCC cells dramatically altered the response of peritoneal macrophages, dendritic cells and tumor-specific $T$ cells. Interestingly, Stat3 was found to directly interact with $14-3-3 \zeta$ and its disruption relieved the inhibition induced by 14-3-3 $\zeta$ in tumor inflammation. Taken together, our studies provide evidence that 14-3-3 $\zeta$ may regulate tumor inflammation and immune response through Stat3 signaling in OSCC.
\end{abstract}

\section{INTRODUCTION}

Head and neck cancer including oral cancer has become the sixth most common cancer worldwide and a notable concern with increasing incidence in young and middle-aged men (Bettendorf et al., 2004; Parkin et al., 1999). It has been reported that oral squamous cell carcinoma (OSCC) is an important component of the worldwide burden of cancer, which bears a 5-year survival rate of approximately $50 \%$ and is poorer than breast cancer or melanoma (Jemal et al., 2011). To explore a promising method of OSCC treatment and prognosis, a

Department of Oral and Maxillofacial Surgery, the First Affiliated Hospital, Zhengzhou University, Zhengzhou 450052, China, 'S School of Stomatology Zhengzhou University, Zhengzhou 450052, China

*Correspondence: xinguanghan@126.com

Received 24 April, 2014; revised 6 November, 2014; accepted 7 November, 2014; published online 30 December, 2014

Keywords: $14-3-3 \zeta$, immune response, oral squamous cell carcinoma, Stat3, tumor inflammation better understanding of the molecular mechanisms behind the development of this disease would be helpful.

14-3-3 is a family of highly conserved proteins that are ubiquitously expressed in various mammalian tissues (Aitken, 1996; Reuther et al., 1996). There are seven isoforms $(\beta, \gamma, \varepsilon, \eta, \sigma, \tau$, and $\zeta$ ) of 14-3-3 encoded in humans (Hashiguchi et al., 2000, Ichimura et al., 1988). Evidence has revealed that dimerized 14-3-3 molecules may exert their function by binding to specific motifs, the most widely accepted of which are RSXpSXP and RXXXpSXP (Lin et al., 2009; Yaffe et al., 1997).

As a pro-oncogenic gene, the expression of $14-3-3 \zeta$ is negatively associated with prognosis in breast cancer patients (Neal et al., 2009). 14-3-3 5 may directly bind to PI3 kinase, promote Akt activation and enhance cell survival (Neal et al., 2012), as well as inactivate tumor suppressors like p53 and p21 (Danes et al., 2008; Wang et al., 2010). Furthermore, Matta et al. demonstrated that $14-3-3 \zeta$ is overexpressed in pre-malignant stages of oral cancer, even in hyperplasia, and that its highexpression is sustained further downstream in the tumorigenic pathway (Matta et al., 2007). Additionally, 14-3-3 proteins have been involved in the regulation of TLR signaling, whereby the stimulation of RAW cells with LPS led to PKC phosphorylation and its association with 14-3-3 $\zeta$ in a MyD88-dependent manner (Faisal et al., 2008). Also, 14-3-3 $\zeta$ was reported to have opposite effects on TLR2 and TLR4 signaling (Schuster et al., 2011), whereby 14-3-3 $\zeta$ inhibited TLR2-mediated NF- $\mathrm{KB}$ activation but enhanced TLR4-mediated NF-KB activation. However, it still remains unclear whether and how 14-3-3 $\zeta$ is involved in tumor inflammation and immune regulation.

The signal transducer and activator of transcription (STAT) family is reported to contain seven members in mammals (Levy et al., 2002; O'Shea et al., 2013). Stat3, the one mostly studied, is implicated in several biological processes. Stat3 may promote tumor growth through its multifunctional role, which includes implicating in cell metastasis, augmenting tumor angiogenesis, and protecting tumors from innate immune recognition (Silver et al., 2004; Wang et al., 2005; Wei et al., 2003). Notably, the Stat3 signaling can be functionally modulated by its interaction with numerous other proteins including $14-3-3 \zeta$ and communicate with various other signaling cascades like the NF- $\kappa \mathrm{B}$, AP-1 or PI-3K pathways (Yu et al., 2007).

The purpose of the present study was to investigate the role of $14-3-3 \zeta$ in tumor inflammation and immune response. In the present study, we found that $14-3-3 \zeta$ is overexpressed in OSCC cell lines and cancer tissues. 14-3-3ל knockdown led to increasing inflammatory cytokine expression, while 14-3-3 $\zeta$ introduction attenuated cytokine production. Moreover, conditioned 
medium from 14-3-3 $\zeta$-disrupted tumor cells triggered increased innate and adaptive immune responses. In addition, 14-3-3 interacts and promotes the nuclear translocation of Stat3 which has been demonstrated to be essential for tumor inflammation.

\section{MATERIALS AND METHODS}

\section{Cell culture and tissue collection}

The human tongue SCC cell line Cal27 was purchased from American Type Culture Collection (ATCC, USA). Cell line Tca8113 was purchased from the Chinese Academy of Sciences (Peking, China). These two cell lines were routinely cultured in RMPI 1640 medium (Gibco, USA) with 10\% fetal calf serum (Gibco, USA) and $1 \%$ penicillin/streptomycin at $37^{\circ} \mathrm{C}$ in an incubator (SANYO, Japan) with $5 \% \mathrm{CO}_{2}$. The medium was changed every 2-3 days and cells were digested using trypsin and were sub-cultured in a new plate when they reached $\sim 80 \%$ confluence. Normal human oral keratinocytes were prepared and maintained as previously described (Min et al., 2004). Briefly, keratinocytes were isolated from human gingival tissue specimens obtained from healthy volunteers $(20-30$ years old) who were undergoing oral surgery. Oral keratinocytes were isolated from separated epithelial tissue by trypsinization, and primary cultures were established in a keratinocyte growth medium containing $0.15 \mathrm{~mm}$ calcium and a supplementary growth-factor bullet kit (KGM; Clonetics, USA). Primary normal human gingival fibroblasts were established from the explant cultures of gingival connective tissue, which was excised from a patient undergoing oral surgery. The cells that proliferated outwardly from the explant culture were continuously cultured in Dulbecco's modified Eagle's medium (DMEM) supplemented with $10 \%$ fetal bovine serum (FBS).

Primary OSCC cells derived from the oral cavity were obtained from surgeries performed at the First Affiliated Hospital of Zhengzhou University and delivered to our laboratory. All adjacent specimens were harvested from morphologically normal appearing tissue located at least $3 \mathrm{~cm}$ from the tumor and were used as the normal control tissue for comparisons with the tumor. Normal mucosal cells were harvested from 21 healthy adults and used as a control. Samples were snapfrozen in liquid nitrogen and stored at $-80^{\circ} \mathrm{C}$ before RNA isolation. The protocols were approved by the Committee for Ethical Procedures of Zhengzhou University, and the study was performed in accordance with the Declaration of Helsinki (2008) for humans.

\section{ELISA analysis}

The levels of inflammatory cytokines in supernatants were evaluated with a commercially available enzyme-linked immunosorbent assay (ELISA; Yanjin Biotechnology Co., China), according to the manufacturer's instructions. The absorbance was read at $450 \mathrm{~nm}$ using a 680XR Microplate reader (Bio-Rad, USA). All of the samples were analyzed in duplicate. The standard curve for protein estimation was made by linear regression analysis.

\section{Adenovirus construction}

All recombinant adenoviruses were constructed according to a previous report (He et al., 1998). Briefly, 14-3-3 $\zeta$ was amplified and sub-cloned into pAdTrack-CMV, an adenoviral shuttle plasmid, whereas GFP was used as a non-specific control. Then, the recombinant shuttle plasmids pAdTrack-CMV and pAdEasy-1 were homologously recombined in the Escherichia coli strain BJ5183. The recombinant plasmids obtained were trans- fected into 293 cells to generate recombinant adenovirus. The virus was amplified and purified, and titers were determined by p24 ELISA kit (Cell Biolabs, Inc., USA), before being stored at $80^{\circ} \mathrm{C}$ for subsequent use.

\section{siRNA transfection}

Scrambled siRNA and small-interfering RNA (siRNA) targeting 14-3-3ל (sc-29583) or Stat3 (sc-29494) were purchased from Santa Cruz Biotechnology (USA). Cells were transfected with scrambled or $14-3-3 \zeta /$ Stat3 siRNA according to the manufacturer's protocol. Briefly, 14-3-3 $\zeta$ or Stat3 and scrambled siRNAs (30 pmol) were diluted in $500 \mu \mathrm{l}$ DMEM and mixed with $5 \mu \mathrm{l}$ Lipofectamine RNAi MAX (Invitrogen, USA). After 15 min of incubation at room temperature, the complexes were added to the cells to a final volume of $3 \mathrm{ml}$ medium. Cells were then harvested at the indicated times for further analysis. The efficiency of $14-3-3 \zeta$ or Stat3 siRNA was confirmed by Western blot analysis of Flag expression.

\section{MTT assay}

$\mathrm{T}$ cell growth and proliferation was evaluated by MTT assay. The experiments were carried out in 96-well plates according to the manufacture's protocols (Roche $\mathrm{GmbH}$, Germany). In the MTT test, tetrazolium salts were transformed by active enzymes of the cells into intracellular formazan deposits and cells were incubated for $4 \mathrm{~h}$ with the tetrazolium salts. After this incubation time, the purple formazan salts formed became soluble. Absorbance was determined at $490 \mathrm{~nm}$.

\section{Reporter gene assays}

OSCC cells were infected with adenovirus-NF-кB-luciferase adenovirus (at $10^{7} \mathrm{pfu} / \mathrm{ml}$ ). Then, 24 and $48 \mathrm{~h}$ after infection, cells were collected and washed with ice-cold PBS, lysed using $250 \mu \mathrm{l}$ Passive Lysis Buffer (Promega, USA), and centrifuged $\left(13,000 \mathrm{rpm}\right.$ for $10 \mathrm{~min}$ at $\left.4^{\circ} \mathrm{C}\right)$. Assays for luciferase activity were performed according to the manufacturer's protocol (Promega) and measured using a luminometer (Veritas; Symantec) and GloMax software (Promega).

\section{Cell fractionation}

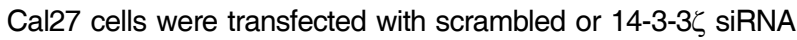
and then harvested at $24 \mathrm{~h}$ after the transfection. Cell fraction was performed with a nuclear and cytoplasmic extraction reagents (Invitrogen, USA) according to the manufacturer's instructions.

\section{Flow cytometry}

Dendritic cells were incubated with the FITC-conjugated specific antibody $\left(60 \mathrm{~min}, 4^{\circ} \mathrm{C}\right)$ and analyzed in a Becton Dickinson FACScan flow cytometer (USA), as described previously (Kwak et al., 2000). At least 100,000 viable cells were analyzed per condition. Data were analyzed using CELLQUEST software (Becton Dickinson).

\section{Immunocytochemistry}

Cal27 cells $\left(5 \times 10^{4}\right)$ were plated on 13-mm glass coverslips coated with poly-L-lysine (Sigma, USA) in $0.5 \mathrm{ml}$ of RMPI 1640 medium (Gibco, USA) with $10 \%$ fetal calf serum per well of a 24-well plate. After overnight incubation, $100 \mathrm{ng}$ of pSVL-STAT3YFP plasmid DNA (Addgene, USA) was co-transfected with 14-

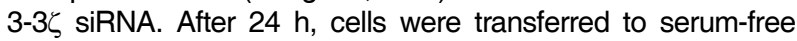
medium and stimulated with $100 \mathrm{ng} / \mathrm{ml}$ leptin for different times. Cells were washed twice with PBS, fixed with $4 \%(\mathrm{v} / \mathrm{v})$ paraformaldehyde for $10 \mathrm{~min}$ at room temperature, and washed 
14-3-3ろ Regulates Immune Response through Stat3 Signaling

Xinguang Han et al.

Table 1. Gene primers for qRT-PCR

\begin{tabular}{lc}
\hline Gene & Sequence \\
\hline 14-3-35 & Forward: 5'-GGA TCC ATG GAT AAA AAT GAG CTG GTT-3' \\
& Reverse: 5'-ACC AGT ATG TAG GCA GTT TTC-3' \\
TNF- $\alpha$ & Forward: 5'-CCCAGGCAGTCAGATCATCTTC-3' \\
& Reverse: 5'-AGCTGCCCCTCAGCTTGA-3' \\
IL-6 & Forward: 5'-GGTACATCCTCGACGGCATCT-3' \\
& Reverse: 5'-GTGCCTCTTTGCTGCTTCAC-3' \\
IFN- $\beta$ & Forward: 5'-GGATGCAGGAAGGAGATCACTG-3' \\
& Reverse: 5'-CGATCCACACGGAGTACTTG-3' \\
RANTES & Forward: 5'-ATATTCCTCCTGGACACCACAC-3' \\
& Reverse: 5'-CACTCCAGCCTGGGGAAGG-3' \\
$\beta$-actin & Forward: 5'-CTCCTTAATGTCACGCAGGATTC-3' \\
& Reverse: 5'-GTGGGGCGCCCCAGGCACCA-3' \\
\hline
\end{tabular}

three times with PBS. After that, cells were observed using an Axiophot fluorescence microscope (Zeiss, Germany).
Quantitative real-time polymerase chain reaction (qRTPCR) analysis

The mRNA of OSCC cell and human tumor tissues was extracted with TRIzol RNA-extraction reagent (Gibco, USA). About $5 \mu \mathrm{g}$ of total RNA for each sample was reversetranscribed into first strand CDNA for qRT-PCR analysis. The qRT-PCR was performed in a final volume of $10 \mu \mathrm{l}$, containing $5 \mu \mathrm{l}$ of SsoFast TM EvaGreen Supermix (BIO-RAD, USA), $1 \mu \mathrm{l}$ of cDNA (1:50 dilution), and $2 \mu \mathrm{l}$ each of the forward and reverse primers $(1 \mathrm{mM})$. The steps in the qRT-PCR were performed as follows: $94^{\circ} \mathrm{C}$ for 2 min for initial denaturation; $94^{\circ} \mathrm{C}$ for $20 \mathrm{~s}, 58^{\circ} \mathrm{C}$ for $15 \mathrm{~s}$, and $72^{\circ} \mathrm{C}$ for $15 \mathrm{~s} ; 2 \mathrm{~s}$ was used for plate reading for 40 cycles; and a melt curve was generated from 65 to $95^{\circ} \mathrm{C}$. The $\beta$-actin was used as a quantitative and qualitative control to normalize the gene expression. Data were analyzed using the formula: $R=2^{-[\Delta \mathrm{Ct} \text { sample- } \Delta \mathrm{Ct} \text { control] }}$. All of the primers used in this experiment are shown in Table 1.

Immunoprecipitation and Western blot analysis

Cells or tumor tissue were homogenized and lysed with RIPA lysis buffer $(100 \mathrm{mM} \mathrm{NaCl}, 50 \mathrm{mM}$ Tris- $\mathrm{HCl}$ pH 7.5, $1 \%$ TritonX100, 1 mM EDTA, $10 \mathrm{mM} \beta$-glycerophosphate, $2 \mathrm{mM}$
A

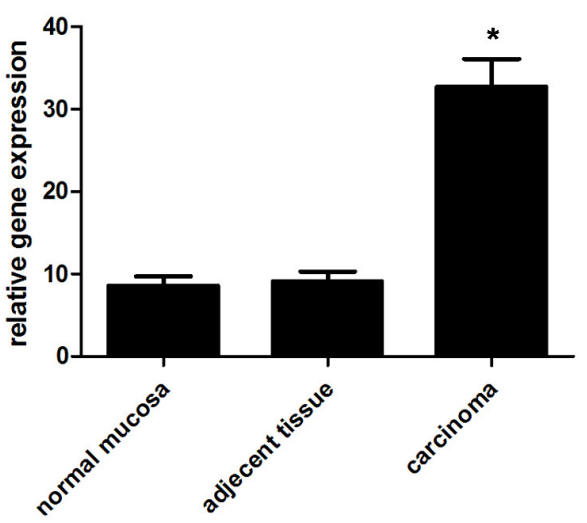

C
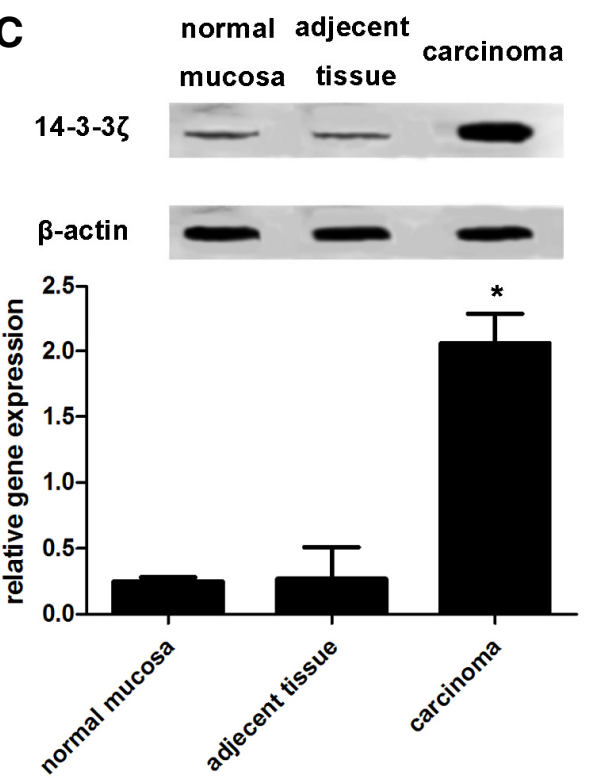

B
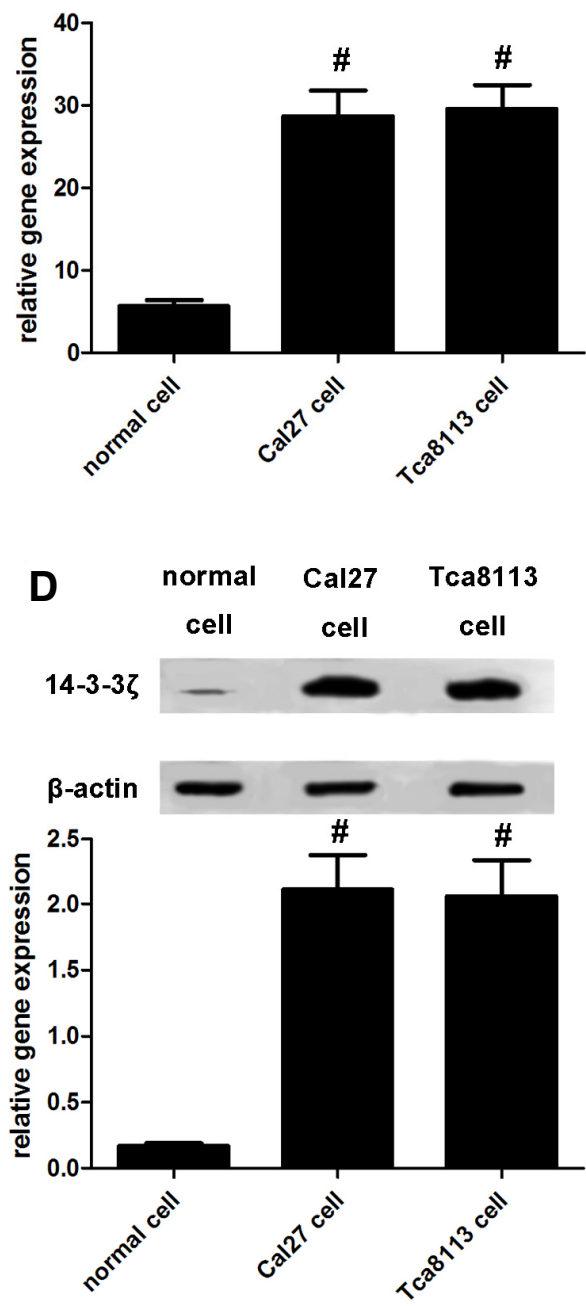

Fig. 1. Analysis of 14-3-3ל expression in oral squamous cell carcinoma (OSCC) cells and cancer tissues. 14-3-3 $\zeta$ expression was analyzed by qRT-PCR in cancer tissues $(A)$ and cell lines (B); Western blot analysis of 14-3$3 \zeta$ expression in cancer tissues (C) and cell lines (D); $\beta$-actin was used as a control. At least three independent experiments were performed. ${ }^{*} p<0.05$ vs. normal cells, \#p $<0.05$ vs. normal mucosa. 
A

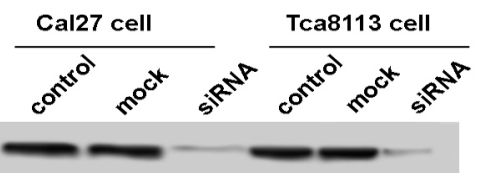

$\beta$-actin

B

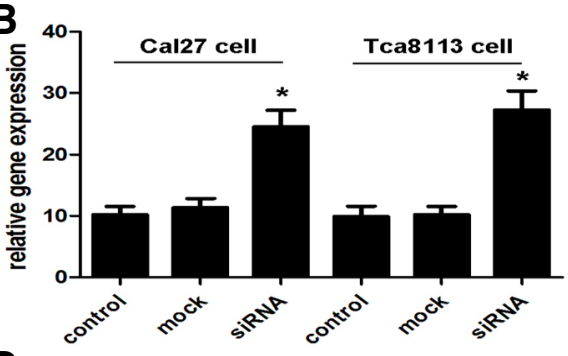

D

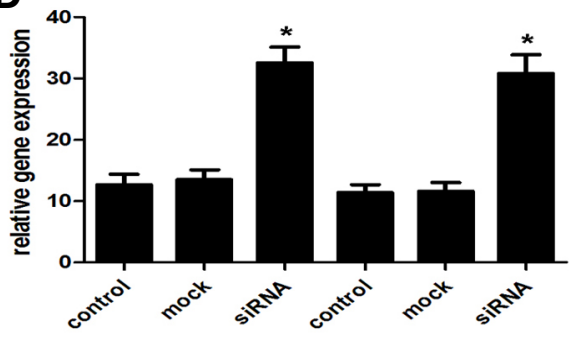

F
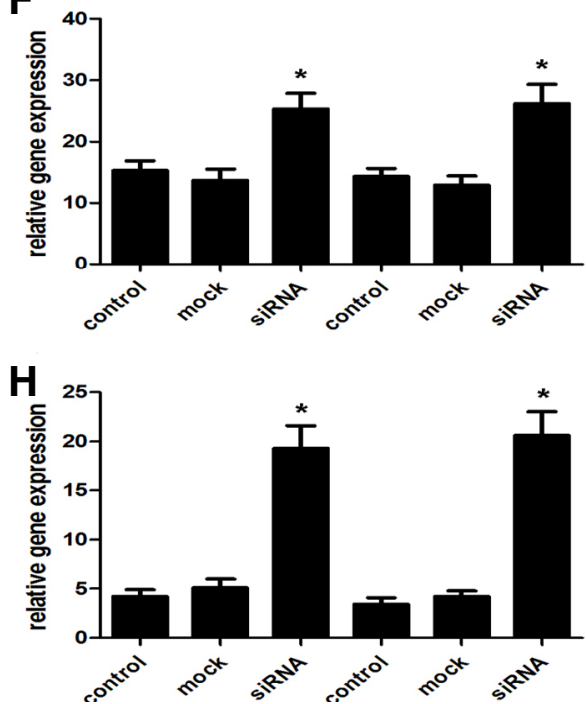

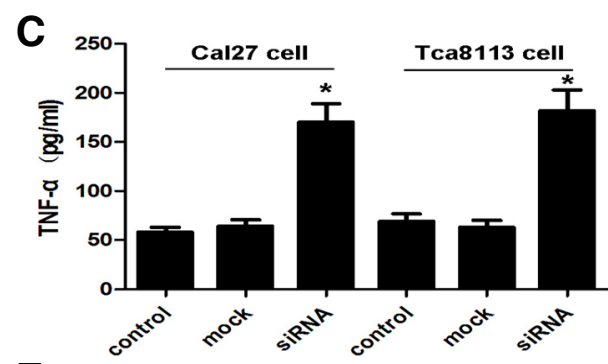

E

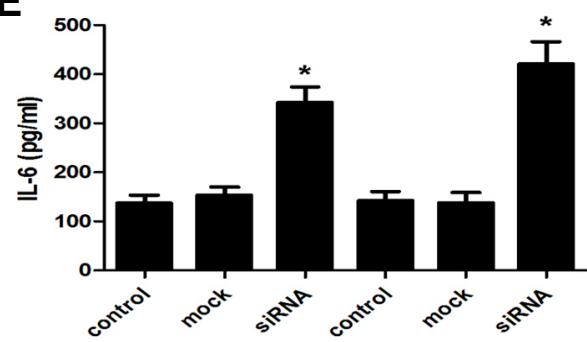

G

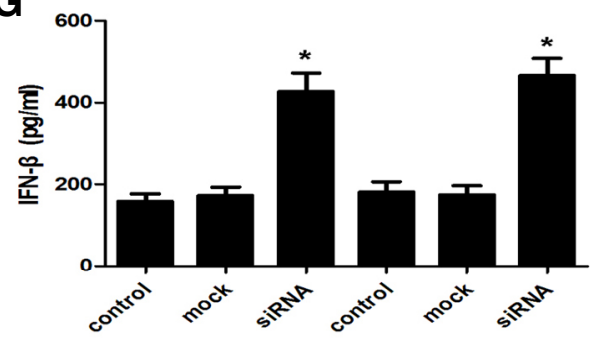

I

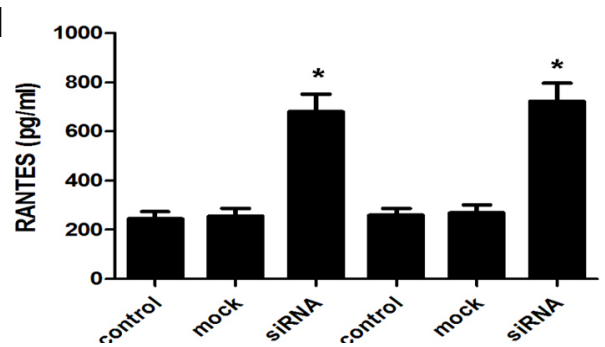

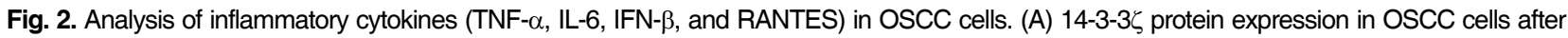
14-3-3 $\zeta$ siRNA transfection; qRT-PCR analysis of TNF- $\alpha(B)$, IL-6 (D), IFN- $\beta$ (F), and RANTES (H) in OSCC cells. Gene expression was normalized to $\beta$-actin mRNA level; Detection of TNF- $\alpha(C)$, IL-6 (E), IFN- $\beta$ (G), and RANTES (I) in OSCC supernatants by ELISA. At least three independent experiments were performed. ${ }^{*} p<0.05$ vs. control.

sodium vanadate and protease inhibitor). Protein concentration was assayed using a micro-BCA protein kit (Pierce, USA). For immunoprecipitation, cell extracts were pre-cleared by Protein A/G Plus beads (Santa Cruz) followed by incubation with primary antibody overnight on a rocker at $4^{\circ} \mathrm{C}$. Immune complexes were pulled-down by incubation with Protein A/G Plus beads for $4 \mathrm{~h}$ at $4^{\circ} \mathrm{C}$ followed by washing twice with lysis buffer containing $0.1 \%$ Triton $X-100$, and twice with lysis buffer without detergent. Bound proteins were eluted by boiling and analyzed by Western blot. For Western blot, $40 \mu \mathrm{g}$ of protein per lane was separated by $12 \%$ SDS-PAGE and electroblotted onto a nitrocellulose membrane (Amersham Pharmacia, Germany). 


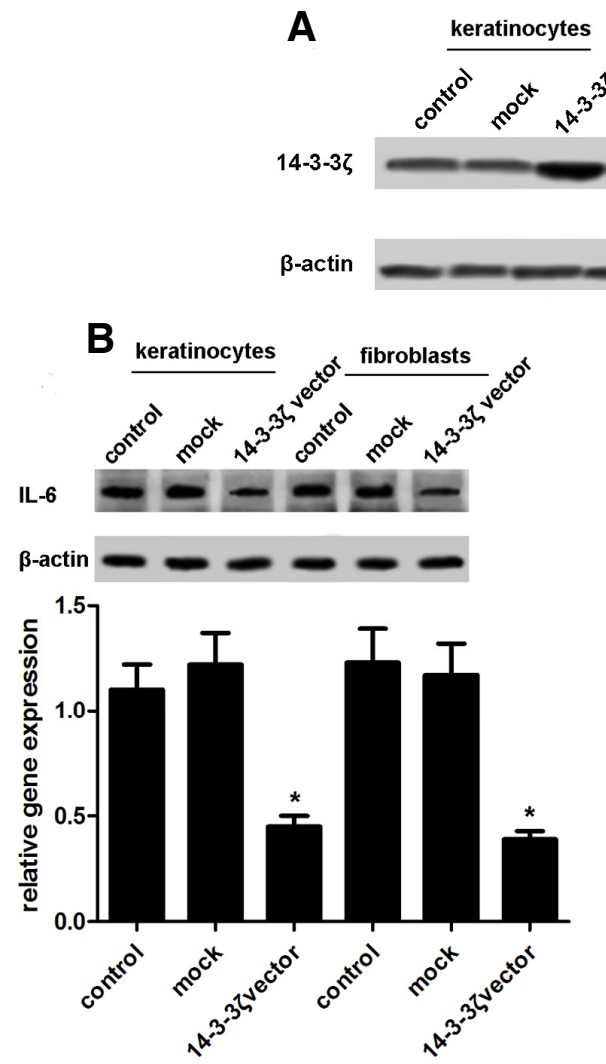

Then, non-specific binding was blocked by incubating with $5 \%$ nonfat milk in Tris-buffered-saline with Tween (TBST; $10 \mathrm{mM}$ Tris- $\mathrm{HCl}, \mathrm{pH}$ of $7.5,150 \mathrm{mM} \mathrm{NaCl}$, and $0.05 \%$ Tween-20) at room temperature for $1 \mathrm{~h}$. Immunodetection of target proteins (14-3-3 total Stat3) and $\beta$-actin was performed using mouse monoclonal antibody (1:1500, Santa Cruz), and anti- $\beta$-actin (Santa Cruz), respectively. Goat anti-mouse IgG (1:5 000, Sigma, USA) followed by enhanced chemiluminescence (ECL, Amersham Pharmacia, USA) was used.

\section{Statistical analysis}

Results are expressed as means \pm SD. Statistical significance was analyzed with one-way factorial ANOVA or Student's twotailed $t$-test. A value of $\mathrm{P}<0.05$ was considered statistically significant. All analyses were conducted using SPSS software (SPSS Inc., USA).

\section{RESULTS}

$14-3-3 \zeta$ is overexpressed in human oral squamous cell carcinoma

To verify the expression of $14-3-3 \zeta$ in oral squamous cell carcinoma, we determined the mRNA level and protein level of 14$3-3 \zeta$ in different cell lines and human tongue cancer tissues. Compared with the normal tissue or normal cells, $14-3-3 \zeta$ was overexpressed in cancer tissue (Figs. $1 \mathrm{~A}$ and $1 \mathrm{C}$ ) and carcinoma cells (Figs. 1B and 1D). These results indicate that 14-3$3 \zeta$ plays an important role in tumor development.
Fig. 3. Analysis of IL-6 and RANTES expression in human normal keratinocytes and gingival fibroblasts. (A)14-3-3ל protein expression in human normal keratinocytes and gingival fibroblasts after 14-3-3 $\zeta$ vector transfection; Western blot analysis of IL-6 (B) and RANTES (C) expression in human normal keratinocytes and gingival fibroblasts after 14-

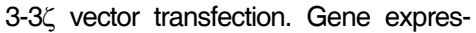
sion was normalized to $\beta$-actin. At least three independent experiments were performed. ${ }^{*} p<0.05$ vs. control.
14-3-3 inhibits proinflammatory cytokine induction The 14-3-3 protein has been reported to inhibit Toll-like receptor (TLR)-mediated proinflammatory cytokine induction (Butt et al., 2012). To investigate whether high expression of $14-3-3 \zeta$ in cancer cell might negatively regulate the expression of proinflammatory cytokines, we generated 14-3-3 $\zeta$ knockdown Cal27

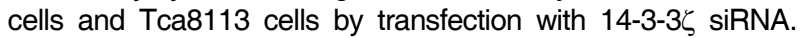
Transfection efficiencies were $20-35 \%$ for Cal27 cells and $10-$ $15 \%$ for Tca8113 cells (Fig. 2A). The mRNA levels of TNF- $\alpha$, IL-6, IFN- $\beta$, and RANTES expression in these cells were analyzed by qRT-PCR. Compared with the control, 14-3-3 $\zeta$ knockdown resulted in a marked increase of TNF- $\alpha$, IL-6, IFN- $\beta$, and RANTES levels in these cells (Figs. 2B, 2D, 2F, and 2H). The results were further confirmed via the detection of TNF- $\alpha$, IL-6, IFN- $\beta$, and RANTES proteins in the supernatants by ELISA (Figs. 2C, 2E, 2G, and 2I). To further verify the role of 14-3-3C in down-regulating the expression of cytokines, we next introduced the 14-3-3 $\zeta$ adenovirus vector into human normal keratinocytes and gingival fibroblasts prior to stimulation by IFN- $\gamma$ ( 50 $\mathrm{U} / \mathrm{ml}$ ) and lipopolysaccharide (LPS; $2.5 \mu \mathrm{g} / \mathrm{ml}$ ). Overexpression of $14-3-3 \zeta$ (Fig. 3A) significantly attenuated IL-6 and RANTES expression in both normal keratinocytes (Fig. 3B) and gingival fibroblasts (Fig. 3C). Overall, these results revealed the inhibitory effect of $14-3-3 \zeta$ on the expression of proinflammatory cytokines.

\section{4-3-3 $\zeta$ regulates innate immune and adaptive immune response}

Compelling evidence has emerged to show that the immune system is constitutively suppressed by established cancers (Lakshmi Narendra et al., 2013). To investigate the role of 14-3- 

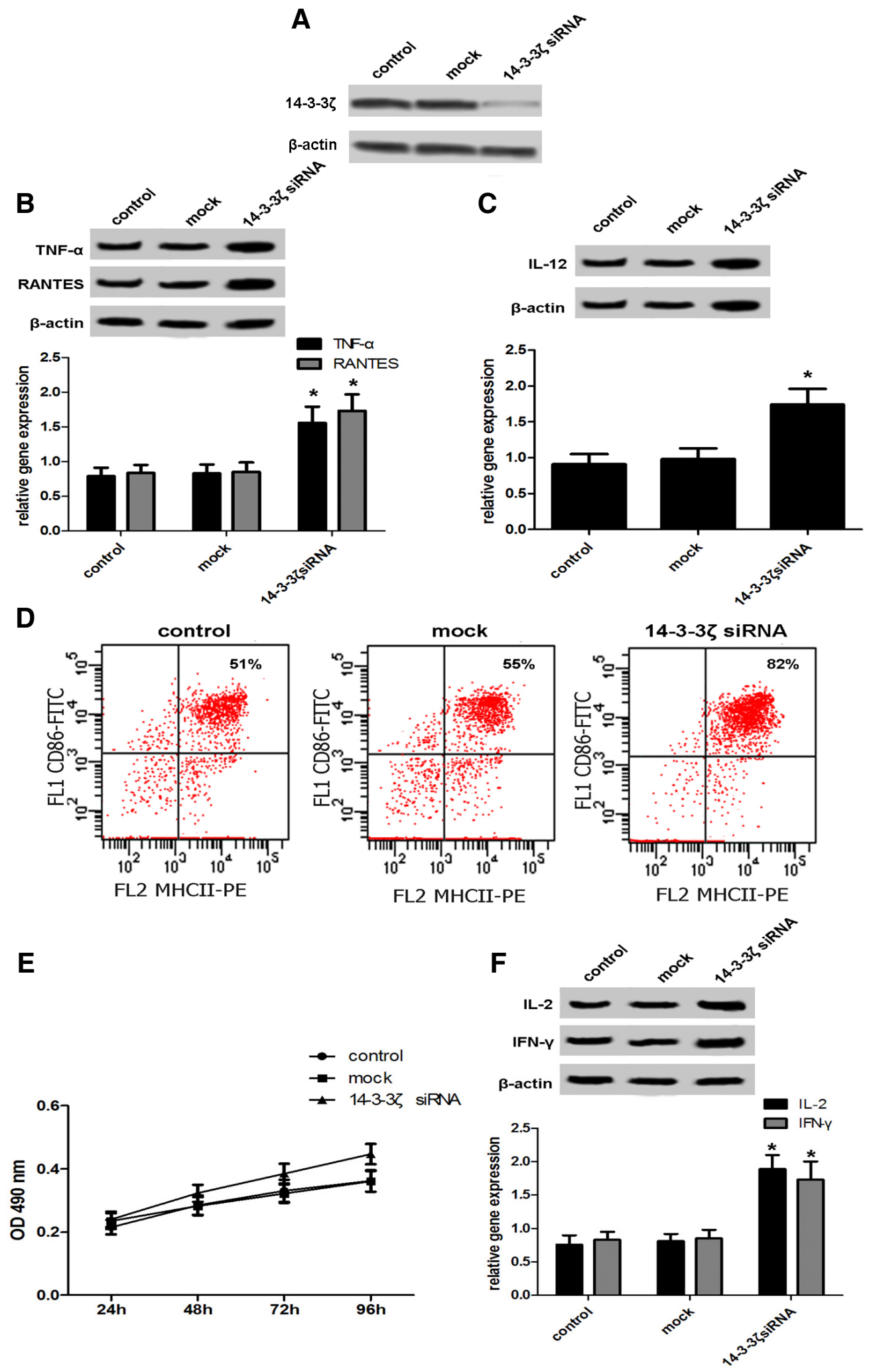

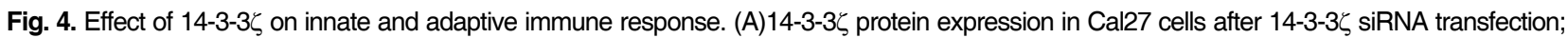
(B) Western blot analysis of TNF- $\alpha$ and RANTES expression in peritoneal macrophages; (C) Western blot analysis of IL-12 expression in dendritic cells; (D) Evaluation of MHC II expression in dendritic cells by flow cytometry; (E) Detection of proliferation rate in tumor antigen-specific T cell by MTT; (F) Western blot analysis of IL-2 and IFN- $\gamma$ expression in tumor antigen-specific T cells; Gene expression was normalized to $\beta$-actin. At least three independent experiments were performed. ${ }^{*} p<0.05$ vs. control. 
14-3-3ろ Regulates Immune Response through Stat3 Signaling

Xinguang Han et al.

A

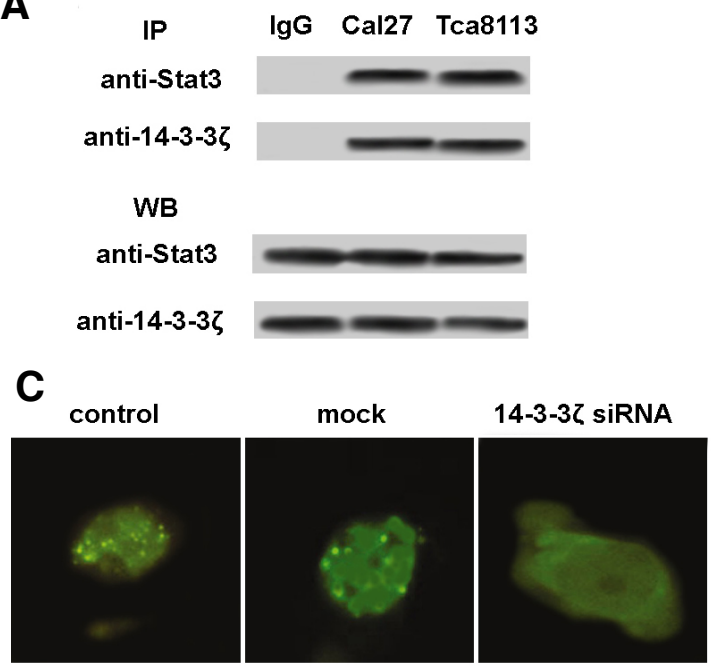

$\mathbf{E}$

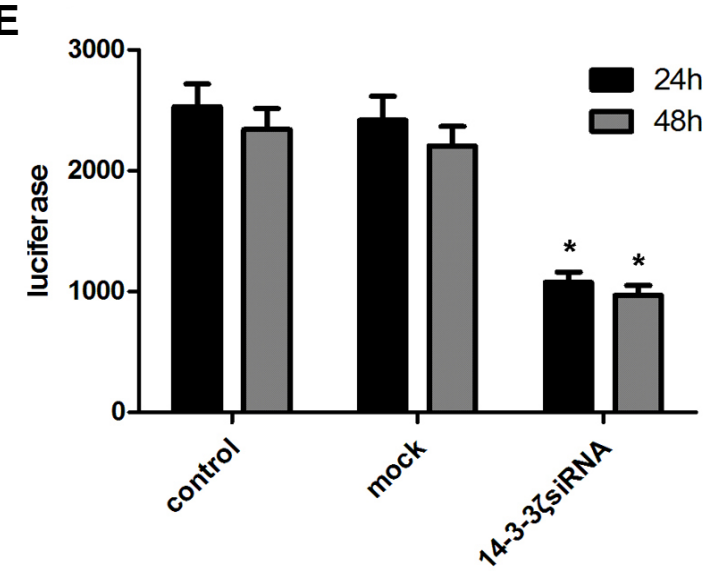

B

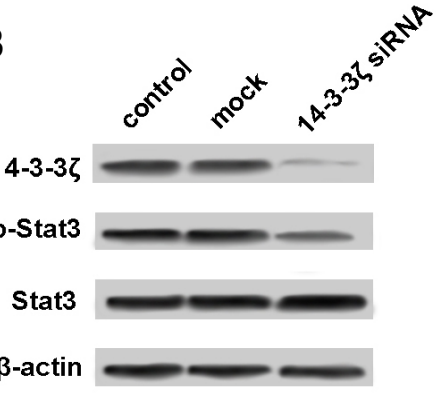

D

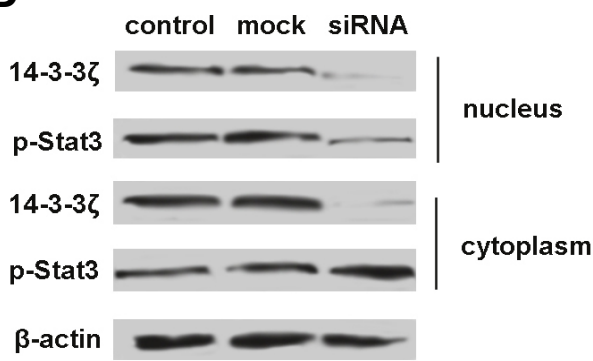

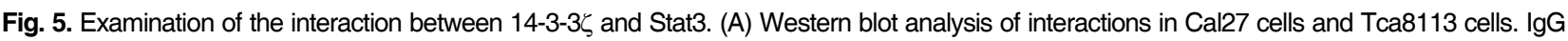
was used as the control for the bait antibody. (B) Detection of total and phosphorylated protein levels of Stat3 by Western blot analysis. Gene expression was normalized to $\beta$-actin. (C) Monitoring Stat3 nuclear translocation by fluorescence microscopy. (D) Cal27 cells were fractionated into cytosolic and nuclear fractions, and equal amounts of protein were analyzed by Western blotting. (E) Evaluation of NF-kB activity in Cal27 cells and Tca8 113 cells. At least three independent experiments were performed. ${ }^{*} p<0.05$ vs. control.

$3 \zeta$ in the regulation of immune response, we disrupted 14-3-3

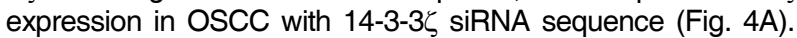
Supernatants from these 14-3-3 $\zeta$-knockdown cells elicited obviously increasing levels of TNF- $\alpha$ and RANTES expression in peritoneal macrophages (Fig. 4B). Dendritic cells are responsible for antigen-presenting in adaptive immune response. Conditioned medium of $14-3-3 \zeta$-knockdown cells also significantly augmented IL-12 (Fig. 4C) and MHC class II (Fig. 4D) expression in dendritic cells. As $T$ cell activation contributes to cell immunity, we next investigated whether interrupting 14-3-3 $\zeta$ in OSCC cells might trigger the activation of tumor antigenspecific T cells. Compared with the control, supernatants from 14-3-3 $\zeta$-disrupted OSCC cells promoted proliferation (Fig. 4E) as well as the IL-2 and IFN-y expression of T cells (Fig. 4F). These results showed that $14-3-3 \zeta$ might negatively regulate innate immune and adaptive immune responses in OSCC.
14-3-3 $\zeta$ interacts with Stat3 and promotes its nuclear translocation

As an important transcription factor, Stat3 is constitutively active in many human cancer cells including OSCC, multiple myeloma (MM) and solid tumors (Bromberg et al., 1999, Wang et al.,

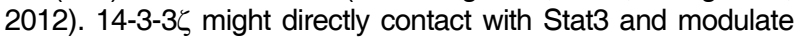
its function. At the same time, the role of Stat3 signaling in the immune regulation of cancer cells has also been elucidated (Wang et al., 2004). To verify whether 14-3-3 $\zeta$ might interact with Stat3 in OSCC, we detected the expected complex using immunoprecipitation. The expression of Stat3 was detected in the 14-3-3 ל immune complex and in Cal27 cells and Tca8113 cell lysate but not in the IgG control (Fig. 5A). Stat3 nuclear translocation was also detected in the 14-3-3 5 -knockdown OSCC (Figs. 5B and 5C). The results showed that the amount of Stat3 distributed in the nucleus was significantly reduced by 14-3-3 $\zeta$ siRNA. The results were confirmed by Western blot 
A

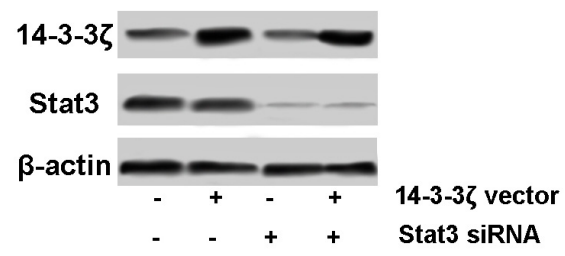

B
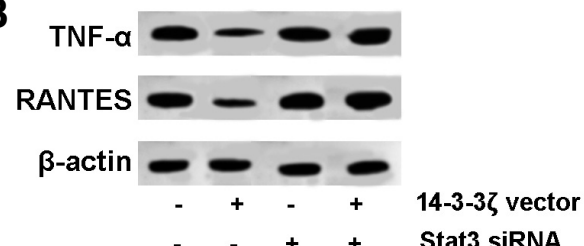

tat3 siRNA

TNF- $\alpha$

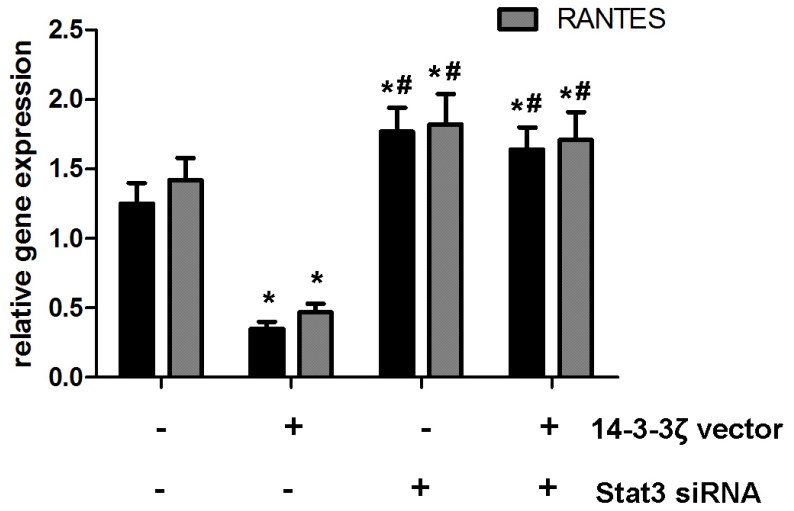

Fig. 6. Detection of TNF- $\alpha$ and RANTES expression in Cal27 cells.

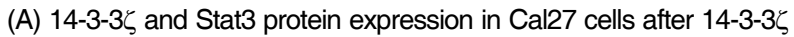
vector or/and Stat3 siRNA transfection; (B) Western blot analysis of TNF- $\alpha$ and RANTES expression in Cal27 cells transfected with 14-3$3 \zeta$ vector or/and Stat3 siRNA. Gene expression was normalized to $\beta$ actin. At least three independent experiments were performed. ${ }^{*} p<$ 0.05 vs. Control, \#p $<0.05$ vs. $14-3-3 \zeta$ vector.

analysis of Stat3 expression in the nucleus and cytoplasm after 14-3-3 $\zeta$ siRNA transfection. Stat3 protein level was reduced in nucleus but was raised in the cytoplasm (Fig. 5D). Furthermore,

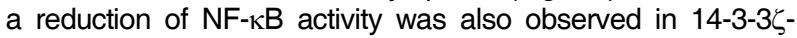
disrupted OSCC cells (Fig. 5E).

\section{Stat3 is essential for the inhibitory role of $14-3-3 \zeta$ in in-}

flammatory cytokine expression

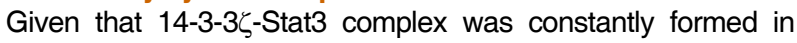
OSCC, we hypothesized that Stat3 might mediate the regulatory effect of $14-3-3 \zeta$ on inflammatory cytokine expression. To test this hypothesis, we constructed three OSCC cell models with the transfection of a 14-3-3 $\zeta$ vector or/and Stat3 siRNA (Fig. 6A). With the established cell models, the expression of TNF- $\alpha$ and RANTES was analyzed by immunoblotting. Compared with the control, overexpression of $14-3-3 \zeta$ prominently down-regulated the expression of TNF- $\alpha$ and RANTES (Fig. $6 \mathrm{~B})$. In contrast, Stat3 knockdown enhanced the protein level of these inflammatory cytokines either with or without the presence of overexpressed $14-3-3 \zeta$ (Fig. 6B). These results indicated that $14-3-3 \zeta$ might regulate inflammatory cytokine expression through Stat3.

\section{DISCUSSION}

Expression of 14-3-3 $\zeta$ has been reported to occur in many types of malignancies, which include lung cancer ( $\mathrm{Li}$ et al., 2008), liver cancer (Choi et al., 2011), uterus, breast carcinoma (Neal et al., 2009), stomach cancer (Jang et al., 2004), head and neck squamous cell carcinoma (Lin et al., 2009) and so on. Notably, the expression of $14-3-3 \zeta$ is elevated in breast cancer, lung cancer, multiple myeloma, glioma, menigioma, esophageal cancer, human head and neck squamous cell carcinomas, prostate tumor, oral cancer, hepatoma and pancreatic adenocarcinoma compared with their nonmalignant counterparts (Yang et al., 2012). Our results found that $14-3-3 \zeta$ was overexpressed in OSCC cell lines and cancer tissues, which confirmed the implication of $14-3-3 \zeta$ in carcinogenesis.

Recently, increasing work has been done to elaborate the mechanism behind tumor cell growth and survival induced by

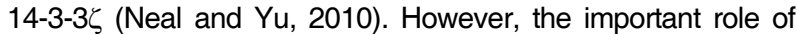
14-3-3 $\zeta$ in the regulation of interactions between tumor cells and the immune system has not been previously reported. Our studies observed that 14-3-3 $\zeta$ knockdown caused elevated proinflammatory cytokine expression in OSCC cell lines. In contrast, introduction of the 14-3-3 $\zeta$ vector into normal cells and fibroblasts led to an inhibition of cytokine expression stimulated by IFN- $\gamma$ and LPS. These results confirmed the effect of 14-3$3 \zeta$ in the modulation of tumor inflammation. Furthermore, conditioned medium from 14-3-3 $\zeta$-knockdown cells up-regulated cytokine synthesis of peritoneal macrophages, boosted the routine activation of dendritic cells and accelerated the normal reaction of tumor-specific $T$ cells. These results revealed the interactions mediated by $14-3-3 \zeta$ between OSCC and the immune system. Our studies also proposed an alternative mechanism, which has been recommended by Ochsenbein et al. (2001), who suggested that established tumors evade immune recognition through either failing to enter lymph nodes or walling themselves off from $T$ cells in lymph nodes.

Activated Stat3 plays a critical role in tumor cell growth, viability, and metastasis in head and neck cancer, as well as in other cancer types (Leeman et al., 2006). Stat3 has been proposed as a therapeutic target for tumor cells as it is a downstream mediator of EGFR and IL6, both of which have been shown to be important in carcinogenesis and angiogenesis (Sriuranpong

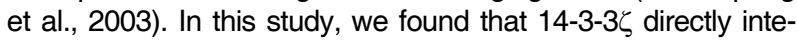
racted with Stat3 and augmented its nuclear translocation. Stat3 signaling activity is tightly regulated by its interacting proteins and multiple signaling cascades and its prolonged activation is associated with various malignancies, including OSCC (Yu et al., 2007). Many studies have indicated that $14-3-3$ proteins are involved in various steps regulating Stat3 activity, including PKC (Saurin et al., 2008), Raf1 (Dhillon et al., 2009), MEK1/2 (Fanger et al., 1998), and PP2A (Sun et al., 2007). Moreover, $14-3-3 \zeta$ may serve as a molecular stabilizer, which contributes to serine phosphorylation and converts Stat3 from the form with lower activity to that with higher activity (Zhang et al., 2012).

Wang et al. (2004) demonstrated that Stat-3 can mediate immune evasion by blocking both the production and sensing of inflammatory signals by multiple components of the immune system. Our studies have shown that cytokine expression inhibition caused by 14-3-3 $\zeta$ was abated by the knockdown of Stat3, which further confirms the hypothesis that Stat3 might play an essential role in the regulation of tumor inflammation.

Collectively, we demonstrated that $14-3-3 \zeta$ regulates tumor inflammation and immune response through Stat3 signaling in 
OSCC. $14-3-3 \zeta$ is overexpressed in OSCC cell lines and cancer tissues. In addition, 14-3-3 $\zeta$ directly interacts with Stat3, which has an essential role in modulating tumor inflammation,

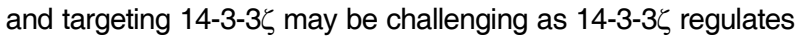
many important proteins that are essential for homeostasis. Thus, more effort should be made to find a promising way for the prevention and treatment of OSCC.

\section{ACKNOWLEDGMENTS}

This study was supported by the grant from department of Oral and Maxillofacial Surgery, the First Affiliated Hospital, Zhengzhou University.

\section{REFERENCES}

Aitken, A. (1996). 14-3-3 and its possible role in co-ordinating multiple signalling pathways. Trends Cell Biol. 6, 341-347.

Bettendorf, O., Piffko, J., and Bankfalvi, A. (2004). Prognostic and predictive factors in oral squamous cell cancer: important tools for planning individual therapy? Oral Oncol. 40, 110-119.

Bromberg, J.F., Wrzeszczynska, M.H., Devgan, G., Zhao, Y., Pestell, R.G., Albanese, C., and Darnell, J.E., Jr. (1999). Stat3 as an oncogene. Cell 98, 295-303.

Butt, A.Q., Ahmed, S., Maratha, A., and Miggin, S.M. (2012). 14-33epsilon and 14-3-3sigma inhibit Toll-like receptor (TLR)-mediated proinflammatory cytokine induction. J. Biol. Chem. 287, 3866538679.

Choi, J.E., Hur, W., Jung, C.K., Piao, L.S., Lyoo, K., Hong, S.W., Kim, S.W., Yoon, H.Y., and Yoon, S.K. (2011). Silencing of 143-3zeta over-expression in hepatocellular carcinoma inhibits tumor growth and enhances chemosensitivity to cis-diammined dichloridoplatium. Cancer Lett. 303, 99-107.

Danes, C.G., Wyszomierski, S.L., Lu, J., Neal, C.L., Yang, W., and Yu, D. (2008). 14-3-3 zeta down-regulates p53 in mammary epithelial cells and confers luminal filling. Cancer Res. 68, 1760-1767.

Dhillon, A.S., Yip, Y.Y., Grindlay, G.J., Pakay, J.L., Dangers, M., Hillmann, M., Clark, W., Pitt, A., Mischak, H., and Kolch, W. (2009). The C-terminus of Raf-1 acts as a 14-3-3-dependent activation switch. Cell. Signal. 21, 1645-1651.

Faisal, A., Saurin, A., Gregory, B., Foxwell, B., and Parker, P.J. (2008). The scaffold MyD88 acts to couple protein kinase Cepsilon to Toll-like receptors. J. Biol. Chem. 283, 18591-18600

Fanger, G.R., Widmann, C., Porter, A.C., Sather, S., Johnson, G.L., and Vaillancourt, R.R. (1998). 14-3-3 proteins interact with specific MEK kinases. J. Biol. Chem. 273, 3476-3483.

Hashiguchi, M., Sobue, K., and Paudel, H.K. (2000). 14-3-3zeta is an effector of tau protein phosphorylation. J. Biol. Chem. 275, 25247-25254.

He, T.C., Zhou, S., da Costa, L.T., Yu, J., Kinzler, K.W., and Vogelstein, B. (1998). A simplified system for generating recombinant adenoviruses. Proc. Natl. Acad. Sci. USA 95, 2509-2514.

Ichimura, T., Isobe, T., Okuyama, T., Takahashi, N., Araki, K., Kuwano, R., and Takahashi, Y. (1988). Molecular cloning of cDNA coding for brain-specific 14-3-3 protein, a protein kinasedependent activator of tyrosine and tryptophan hydroxylases. Proc. Natl. Acad. Sci. USA 85, 7084-7088.

Jang, J.S., Cho, H.Y., Lee, Y.J., Ha, W.S., and Kim, H.W. (2004). The differential proteome profile of stomach cancer: identification of the biomarker candidates. Oncol. Res. 14, 491-499.

Jemal, A., Bray, F., Center, M.M., Ferlay, J., Ward, E., and Forman, D. (2011). Global cancer statistics. CA Cancer J. Clin. 61, 69-90.

Kwak, B., Mulhaupt, F., Myit, S., and Mach, F. (2000). Statins as a newly recognized type of immunomodulator. Nat Med. 6, 13991402

Lakshmi Narendra, B., Eshvendar Reddy, K., Shantikumar, S., and Ramakrishna, S. (2013). Immune system: a double-edged sword in cancer. Inflamm. Res. 62, 823-834.

Leeman, R.J., Lui, V.W., and Grandis, J.R. (2006). STAT3 as a therapeutic target in head and neck cancer. Expert Opin. Biol. Ther. 6, 231-241.

Levy, D.E., and Darnell, J.E., Jr. (2002). Stats: transcriptional control and biological impact. Nat. Rev. Mol. Cell Biol. 3, 651662.
Li, Z., Zhao, J., Du, Y., Park, H.R., Sun, S.Y., Bernal-Mizrachi, L., Aitken, A., Khuri, F.R., and Fu, H. (2008). Down-regulation of 14-3-3zeta suppresses anchorage-independent growth of lung cancer cells through anoikis activation. Proc. Natl. Acad. Sci. USA 105, 162-167.

Lin, M., Morrison, C.D., Jones, S., Mohamed, N., Bacher, J., and Plass, C. (2009). Copy number gain and oncogenic activity of YWHAZ/14-3-3zeta in head and neck squamous cell carcinoma. Int. J. Cancer 125, 603-611.

Matta, A., Bahadur, S., Duggal, R., Gupta, S.D., and Ralhan, R. (2007). Over-expression of 14-3-3zeta is an early event in oral cancer. BMC Cancer 7, 169.

Min, B.M., Lee, G., Kim, S.H., Nam, Y.S., Lee, T.S., and Park, W.H (2004). Electrospinning of silk fibroin nanofibers and its effect on the adhesion and spreading of normal human keratinocytes and fibroblasts in vitro. Biomaterials 25, 1289-1297.

Neal, C.L., and Yu, D. (2010). 14-3-3zeta as a prognostic marker and therapeutic target for cancer. Expert Opin. Ther. Targets 14, 1343-1354.

Neal, C.L., Yao, J., Yang, W., Zhou, X., Nguyen, N.T., Lu, J., Danes, C.G., Guo, H., Lan, K.H., Ensor, J., et al. (2009). 14-3-3zeta overexpression defines high risk for breast cancer recurrence and promotes cancer cell survival. Cancer Res. 69, 3425-3432.

Neal, C.L., Xu, J., Li, P., Mori, S., Yang, J., Neal, N.N., Zhou, X., Wyszomierski, S.L., and Yu, D. (2012). Overexpression of 14-33zeta in cancer cells activates PI3K via binding the p85 regulatory subunit. Oncogene 31, 897-906.

O'Shea, J.J., Holland, S.M., and Staudt, L.M. (2013). JAKs and STATs in immunity, immunodeficiency, and cancer. N. Engl. J. Med. 368, 161-170.

Ochsenbein, A.F., Sierro, S., Odermatt, B., Pericin, M., Karrer, U., Hermans, J., Hemmi, S., Hengartner, H., and Zinkernagel, R.M. (2001). Roles of tumour localization, second signals and cross priming in cytotoxic T-cell induction. Nature 411, 1058-1064.

Parkin, D.M., Pisani, P., and Ferlay, J. (1999). Estimates of the worldwide incidence of 25 major cancers in 1990. Int. J. Cancer $80,827-841$.

Reuther, G.W., and Pendergast, A.M. (1996). The roles of 14-3-3 proteins in signal transduction. Vitam. Horm. 52, 149-175.

Saurin, A.T., Durgan, J., Cameron, A.J., Faisal, A., Marber, M.S., and Parker, P.J. (2008). The regulated assembly of a PKCepsilon complex controls the completion of cytokinesis. Nat. Cell Biol. 10, 891-901.

Schuster, T.B., Costina, V., Findeisen, P., Neumaier, M., and AhmadNejad, P. (2011). Identification and functional characterization of 14-3-3 in TLR2 signaling. J. Proteome Res. 10, 4661-4670.

Silver, D.L., Naora, H., Liu, J., Cheng, W., and Montell, D.J. (2004). Activated signal transducer and activator of transcription (STAT) 3: localization in focal adhesions and function in ovarian cancer cell motility. Cancer Res. 64, 3550-3558.

Sriuranpong, V., Park, J.I., Amornphimoltham, P., Patel, V., Nelkin, B.D., and Gutkind, J.S. (2003). Epidermal growth factor receptorindependent constitutive activation of STAT3 in head and neck squamous cell carcinoma is mediated by the autocrine/paracrine stimulation of the interleukin 6/gp130 cytokine system. Cancer Res. 63, 2948-2956.

Sun, L., Stoecklin, G., Van Way, S., Hinkovska-Galcheva, V., Guo, R.F., Anderson, P., and Shanley, T.P. (2007). Tristetraprolin (TTP)-14-3-3 complex formation protects TTP from dephosphorylation by protein phosphatase $2 \mathrm{a}$ and stabilizes tumor necrosis factor-alpha mRNA. J. Biol. Chem. 282, 3766-3777.

Wang, T., Niu, G., Kortylewski, M., Burdelya, L., Shain, K., Zhang, S., Bhattacharya, R., Gabrilovich, D., Heller, R., Coppola, D., et al. (2004). Regulation of the innate and adaptive immune responses by Stat-3 signaling in tumor cells. Nat. Med. 10, 4854.

Wang, M.L., Shin, M.E., Knight, P.A., Artis, D., Silberg, D.G., Suh, E., and Wu, G.D. (2005). Regulation of RELM/FIZZ isoform expression by $\mathrm{Cdx} 2$ in response to innate and adaptive immune stimulation in the intestine. Am. J. Physiol. Gastrointest Liver Physiol. 288, G1074-1083.

Wang, B., Liu, K., Lin, H.Y., Bellam, N., Ling, S., and Lin, W.C. (2010). 14-3-3Tau regulates ubiquitin-independent proteasomal degradation of p21, a novel mechanism of p21 downregulation in breast cancer. Mol. Cell. Biol. 30, 1508-1527.

Wang, X., Crowe, P.J., Goldstein, D., and Yang, J.L. (2012). STAT3 
inhibition, a novel approach to enhancing targeted therapy in human cancers (review). Int. J. Oncol. 41, 1181-1191.

Wei, D., Le, X., Zheng, L., Wang, L., Frey, J.A., Gao, A.C., Peng, Z., Huang, S., Xiong, H.Q., Abbruzzese, J.L., et al. (2003). Stat3 activation regulates the expression of vascular endothelial growth factor and human pancreatic cancer angiogenesis and metastasis. Oncogene 22, 319-329.

Yaffe, M.B., Rittinger, K., Volinia, S., Caron, P.R., Aitken, A., Leffers, H., Gamblin, S.J., Smerdon, S.J., and Cantley, L.C. (1997). The structural basis for 14-3-3:phosphopeptide binding specificity. Cell 91, 961-971.
Yang, X., Cao, W., Zhang, L., Zhang, W., Zhang, X., and Lin, H. (2012). Targeting 14-3-3zeta in cancer therapy. Cancer Gene Ther. 19, 153-159.

Yu, H., Kortylewski, M., and Pardoll, D. (2007). Crosstalk between cancer and immune cells: role of STAT3 in the tumour microenvironment. Nat. Rev. Immunol. 7, 41-51.

Zhang, J., Chen, F., Li, W., Xiong, Q., Yang, M., Zheng, P., Li, C., Pei, J., and Ge, F. (2012). 14-3-3zeta interacts with stat3 and regulates its constitutive activation in multiple myeloma cells. PLoS One 7, e29554. 